INTERNATIONAL DESIGN CONFERENCE - DESIGN 2018

https://doi.org/10.21278/idc.2018.0537

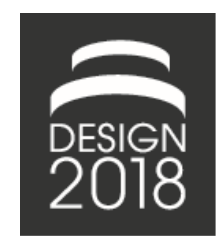

\title{
DESIGN AUTOMATION STATE OF PRACTICE - POTENTIAL AND OPPORTUNITIES
}

\author{
E. Rigger and T. Vosgien
}

\begin{abstract}
Design Automation has been in focus of research and application for several decades. This paper aims at establishing the current view of design automation and identification of potential for adoption based on a survey conducted in German speaking countries and a hypothesis based multivariate analysis based on networks. The findings show that design automation is still considered a means of automation of repetitive design tasks and potential for enhanced application exists. The necessity for methods supporting designers for identification and definition of design automation tasks is urged.
\end{abstract}

Keywords: knowledge based engineering (KBE), computational design methods, computational design synthesis, design automation, design practice

\section{Introduction}

Despite significant research in the field of design automation, the industrial application most often limits to either configuration systems (Zhang, 2014; Willner et al., 2016) or automation of routine design tasks within product development (Verhagen et al., 2012). Reasons for this may list the lack of knowledge and understanding of practitioners and software vendors with respect to advanced design automation methods that are currently available in the academia (Bolognini et al., 2012). Thus, state of practice does not reflect the full potential of available design automation methods and tools. In order to investigate design automation state of practice and determine potential for further application of design automation in industry, this paper aims at establishing the current view how the industry perceives the availability, potential and the purpose of design automation. Therefore, a survey is conducted in central European German speaking countries for collecting data targeting the extent of design automation knowledge, experience, drivers as well as requirements related to the product complexity, the multidisciplinary nature of product development and the advent usage of numerical simulations. A hypothesis-driven multivariate analysis (Blessing and Chakrabarti, 2009) is conducted on the collected data, including network analyses in order to qualitatively investigate impacts and correlations within the data sets and based thereon, derive conclusion with regards to current state of practice as well as prevalent potential for design automation application.

Whereas Chapter 2 presents relevant studies addressing the assessment of design automation application in industry, Chapter 3 introduces the pursued methodology including the methods used for the survey as well as for post-processing the results of this survey. Chapter 4 presents the results yielded and within Chapter 5 the corresponding findings are discussed and analysed. Finally, Chapter 6 provides concluding remarks and outlines future research.

\section{Background}

In an industrial survey, Cederfeldt and Elgh (2005) conducted structured interviews with eleven participants from Swedish manufacturing Small and Medium Enterprises (SME) and applied both 
open questions as well as multiple-choice questionnaires. The need for design automation is determined based on a predefined list of drivers and design automation potential is analysed based on questions related to both process and product maturity with respect to the prevalent level of formalization and types of processes. However, the responses obtained were not put in context with the background of the responders to indicate the importance of drivers in a particular context and thereby determine correlations within data sets. Further, whereas the provided list of drivers accounts for drivers related to process time, product performance and resources it does not consider aspects related to design process support such as enabling of collaborative design as reported by Hvam et al. (2006). Based on the drivers determined by Cederfeldt and Elgh (2005), Rigger et al. (2016) investigated design automation literature with respect to reported benefits. Their findings showed that the following qualitative benefits are mostly used for justifying benefits of design automation application in literature:

- Establishment of knowledge base, through formalization of product and process knowledge,

- Generation of performance efficient designs using optimization methods;

- Automation of laborious design tasks, i.e. automation of repetitive and complex design tasks, e.g. including large numbers of design variables and highly constrained design spaces

- Generation of design alternatives to support the ideation process,

- Lead time minimization,

- Quality assurance, for reduction of errors in design and development of validated designs.

In order to provide an overview what type of design tasks design automation can be applied to, Rigger et al. (2018) present a comprehensive design automation task categorization for which the design automation methods were developed for. Thereby, the authors aim at consolidation of the two major design automation research fields, namely Knowledge-Based Engineering (KBE) and Computational Design Synthesis (CDS) that both investigate computational approaches supporting design activities by means of automation. KBE addresses the automation of routine design activities and commercial software solutions exist (Chandrasegaran et al., 2013) which are usually advertised with time and cost savings associated to automation of repetitive activities, e.g. Dassault Systems (2010). On the other hand, CDS aims at supporting the early design stages with algorithmic approaches for design space modelling for the generation of alternative solutions as well as solving of complex design tasks, however, only few industrial applications of CDS methods have been reported (Rigger et al., 2018). Whereas a comprehensive overview of state of the art design automation is presented and opportunities for design automation application are highlighted, the state of practice of industry still needs to be examined in detail by means of establishing the current view of design automation with respect to drivers and potential in industry. In order to obtain a holistic view of design automation practice and potential in industry, correlations of drivers and meta-data such as company size, product development processes applied etc. need to be investigated so to determine specific requirements according to dimensions such as product types, company size, the engineering context and the experience of the participant.

\section{Method}

This section first introduces a set of hypotheses to predict correlations of design automation state of practice and drivers, respectively, as well as the dimensions:

- company size,

- rate of repetitiveness in design,

- type of products,

- complexity of products, and already prevalent

- design automation experience of participants.

The characteristics of the pursued online survey/questionnaire method for data collection are introduced before the details of the multivariate analysis are presented within Section 3.3 so to enable verification of the hypotheses and correlations in the datasets. 


\subsection{Design automation hypotheses}

For structuring the data collection and analysis, following hypotheses are stated so to determine need and potential for design automation application in industrial practice:

1. "SMEs generally don't apply design automation". This hypothesis allows to further sharpen the observations by (Cederfeldt and Elgh, 2005), who reported a varying state of design automation in SMEs.

2. Design automation is applied for the automation of repetitive activities in adaptive design", so to assess whether design automation is usually considered from a KBE perspective (Verhagen et al., 2012).

Following hypotheses aim at investigation of impacts stemming from design type, product complexity and design automation experience. Whereas hypotheses 3 and 4 permit the analysis of specific needs of practitioners with respect to the type of product they design, hypothesis 5 aims at investigation of the general satisfaction with already implemented design automation solutions in practice:

3. "The viewpoint on design automation drivers correlates with the type of design applied."

4. "The more complex the product, the higher the need for design automation aiming at support for error reduction and solving of complex design tasks."

5. "Design automation experience positively impacts the drivers for further design automation application."

\subsection{Data collection}

In order to keep the bias as low as possible, an online survey was selected as the method of choice to collect the necessary data. Further, to not advocate any particular type of design automation, no explicit definition of design automation was provided. Table 1 lists the relevant questions of the survey including the questions and the related set of answers provided in the online form.

Table 1. Questions and possible answers as used within the online survey

\begin{tabular}{|c|l|l|}
\hline$\#$ & Question & \multicolumn{1}{|c|}{ Possible answer(s) } \\
\hline 1 & Which type of design do you apply in your daily business? * & $\begin{array}{l}\text { a) variant design; b) adaptive design } \\
\text { c) original design; d) something else... }\end{array}$ \\
\hline 2 & What systems are currently in use for product development? & $\begin{array}{l}\text { ERP, PDM, CAD, CAM, CAE, CAPP, } \\
\text { CRM, PPS, none of these }\end{array}$ \\
\hline 3 & $\begin{array}{l}\text { What is the relevance of software implementation in product } \\
\text { development? }\end{array}$ & $\begin{array}{l}\text { very important, important, not so } \\
\text { important, not important }\end{array}$ \\
\hline 4 & Are there multiple disciplines involved in design? & always, often, occasionally, rarely, never \\
\hline 5 & Is the application of numeric simulations required? & always, often, occasionally, rarely, never \\
\hline 6 & What is the rate of repetitive tasks in design? & $\ldots$ \\
\hline 7 & Have you heard about design automation? & yes, no \\
\hline 8 & Do you have experience with design automation? & yes, no \\
\hline 9 & $\begin{array}{l}\text { What is the relevance of design automation implementation } \\
\text { in product development? }\end{array}$ & $\begin{array}{l}\text { very important, important, not so } \\
\text { important, not important }\end{array}$ \\
\hline 10 & $\begin{array}{l}\text { What is the relevance of the following drivers for design } \\
\text { automation implementation? (drivers are listed in Figure 7) }\end{array}$ & $\begin{array}{l}\text { for each driver: very important, } \\
\text { important, not so important, not } \\
\text { important }\end{array}$ \\
\hline 11 & What is the number of employees worldwide? & $\ldots$ \\
\hline
\end{tabular}

* The definitions of the types of design refer to Pahl et al. (2007).

The set of participants was limited to established company databases available to us. The surveyed companies have the following features:

- They are located in Austria, Germany and in the German-speaking part of Switzerland,

- They are categorized as mechanical engineering companies,

- They employ more than 80 people. 
Thus, a set of 146, 1150, and 195 potential participants from Austrian, German and Swiss companies is yielded, respectively. The sample design based on companies located in German speaking countries can be justified by the fact that these countries represent a highly industrialized region of Europe which can be considered representative with respect to high-value added manufacturing companies.

\subsection{Network analysis}

In order to assess interrelations and impacting factors as listed within the set of hypotheses listed in Section 3.1, this paragraph introduces the network method that is applied to visualize relational information of the data sets obtained from the survey and enable both qualitative and quantitative analysis. In this respect, quantitative denotes the network characteristic of edge length as used for calculations of the networks for hypotheses 3-5. For the actual computation and visualization of networks, Wolfram Mathematica 11.2 is used; in particular the implementation of the "SpringElectricalEmbedding" method is applied. "SpringElectricalEmbedding" is a force directed algorithm that aims at minimizing mechanical and electrical energy assuming each edge corresponds to a spring and each vertex has a charge. A force directed algorithm is selected because it is particularly useful to minimize edge crossings, highlight symmetry and maximize the distance between non-adjacent nodes which supports the identification of clusters within networks (Quigley, 2001). Further, force directed algorithms permit the layout generation for weighted graphs as used for the analysis of hypotheses 3-5. With this respect, weighted graphs are calculated as follows: an edge is created between the type of design (hypothesis 3), rate of simulations/disciplines (both hypothesis 4), the prevalent experience (hypothesis 5) and each of the design automation drivers. Next, the edge weight is calculated based on the rating of drivers according to the following formula:

$$
\text { Weight }=1+\frac{n_{v i} * w_{v i}+n_{i} * w_{i}+n_{n s i} * w_{n s i}+n_{n i} * w_{n i}}{\text { NumberOfAnswers }} ; w=\left\{\begin{array}{c}
w_{v i} \\
w_{i} \\
w_{n i i} \\
w_{n i}
\end{array}\right\}=\left\{\begin{array}{c}
-.75 \\
-.5 \\
.75
\end{array}\right\}
$$

$n_{v i}, n_{i}, n_{n s i}$ and $n_{n i}$ correspond to the number of responses who rated the driver very important, important, not so important and not important, respectively. Similarly, $w_{v i}, w_{i}, w_{n s i}$, and $w_{n i}$ correspond to the weighting factors associated to a rating answer "very important", "important", "not so important" and "not important", respectively.

\section{Results}

The online survey yielded a rate of responses of up to 47 participants. Considering a pool of 1491 possible participants and a confidence level of $95 \%$, the margin of error is calculated as $15 \%$ (Tanur, 2011). Thus, the results of this survey do not permit to validate or contradict the hypotheses listed in Section 3, but rather to reassert or weaken them. In the following, Section 4.1 presents the results yielded by the survey and the response rate per question. Within Section 4.2, networks are generated in order to qualitatively identify correlating factors and to quantitatively weight the resulted drivers and their correlating factors.

\subsection{Linear analysis}

With respect to the online survey, a total number (N) up to 47 participants was attained and $33 \%$ of participants are employed by SMEs with less than 250 employees. In the following, the results of the questionnaire, as defined in Table 1, are successively introduced. The rate of response per question is indicated in the caption of the corresponding figure. Figure 1 shows the percentage of users applying one specific type of design. It has to be noted that multiple answers are valid, e.g. a company can apply both variant design and original design since the boundaries of this classification are often not precise (Pahl et al., 2007). Whereas Figure 2 shows the rating of current application of selected software applications in product development, Figure 3 indicates whether participants (a) have ever heard about design automation, and, (b), whether they have already gained practical experience applying design automation. 


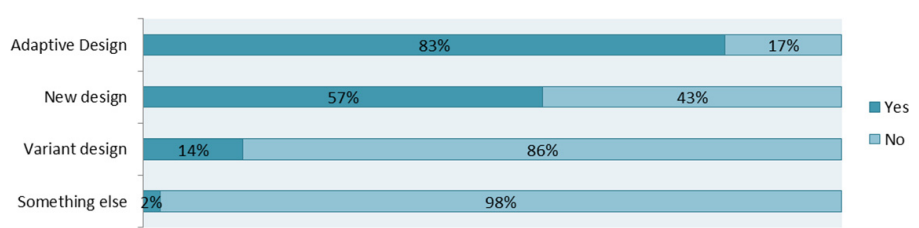

Figure 1. Which type of design do you apply in your daily business? $(\mathrm{N}=42)$

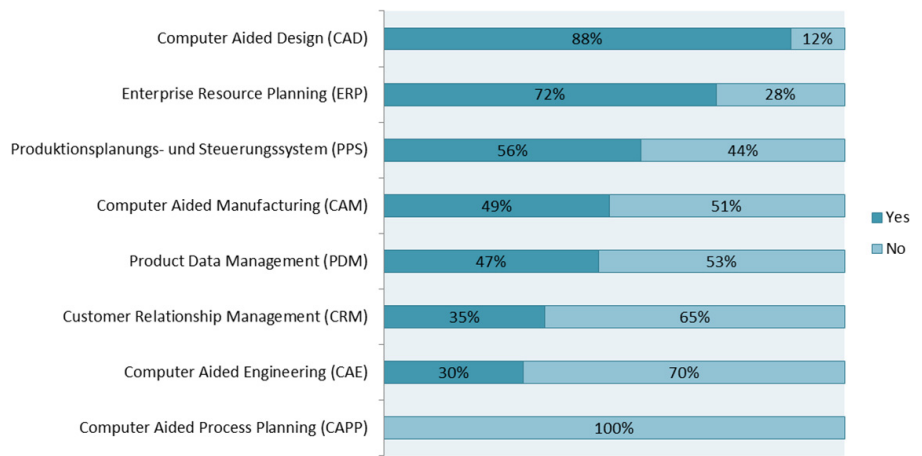

Figure 2. What systems are currently in use for product development? $(\mathrm{N}=42)$

(a)

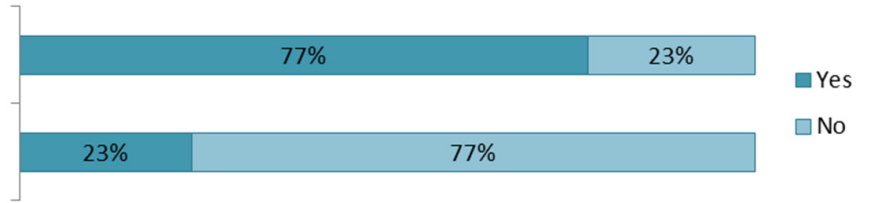

Figure 3. (a) Have you heard about design automation? $(N=44)$; (b) Do you have experience with design automation? $(\mathrm{N}=47)$

Figure 4 shows the rating of relevance of (a) software support for product development in general and (b) specific to design automation. With respect to product design complexity, the multi-disciplinary functional requirements and the various behavioural scenarios are handled and verified with the use of numerical simulations (Shephard et al., 2004). Therefore, in order to rate this complexity, Figure 5 assesses the frequency of (a) involvement of multiple disciplines in design, and, (b), the rate of application of advanced numerical simulations in design. Figure 6 introduces the rate of repetitiveness in design and Figure 7 presents the set of rated drivers in decreasing order, i.e. starting with the highest ranked driver at the top to the least rated driver at the very bottom of the list.

(a)

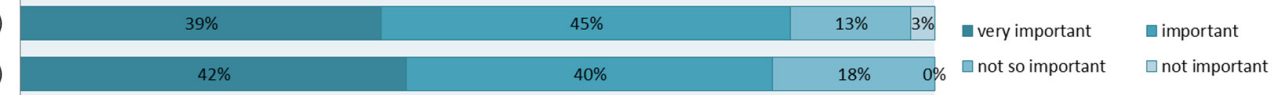

Figure 4. (a) What is the relevance of software implementation in product development? $(\mathrm{N}=45)$; (b) What is the relevance of design automation implementation in product development? $(\mathrm{N}=38)$

(a)

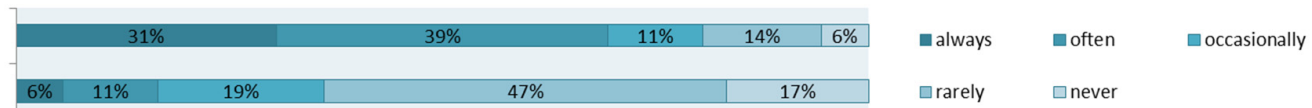

Figure 5. (a) Are there multiple disciplines involved in design? $(\mathrm{N}=36)$; (b) Is the application of numeric simulations required? $(\mathrm{N}=36)$ 


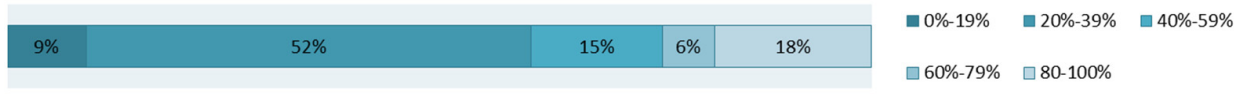

Figure 6. What is the rate of repetitive tasks in design? $(\mathrm{N}=33)$

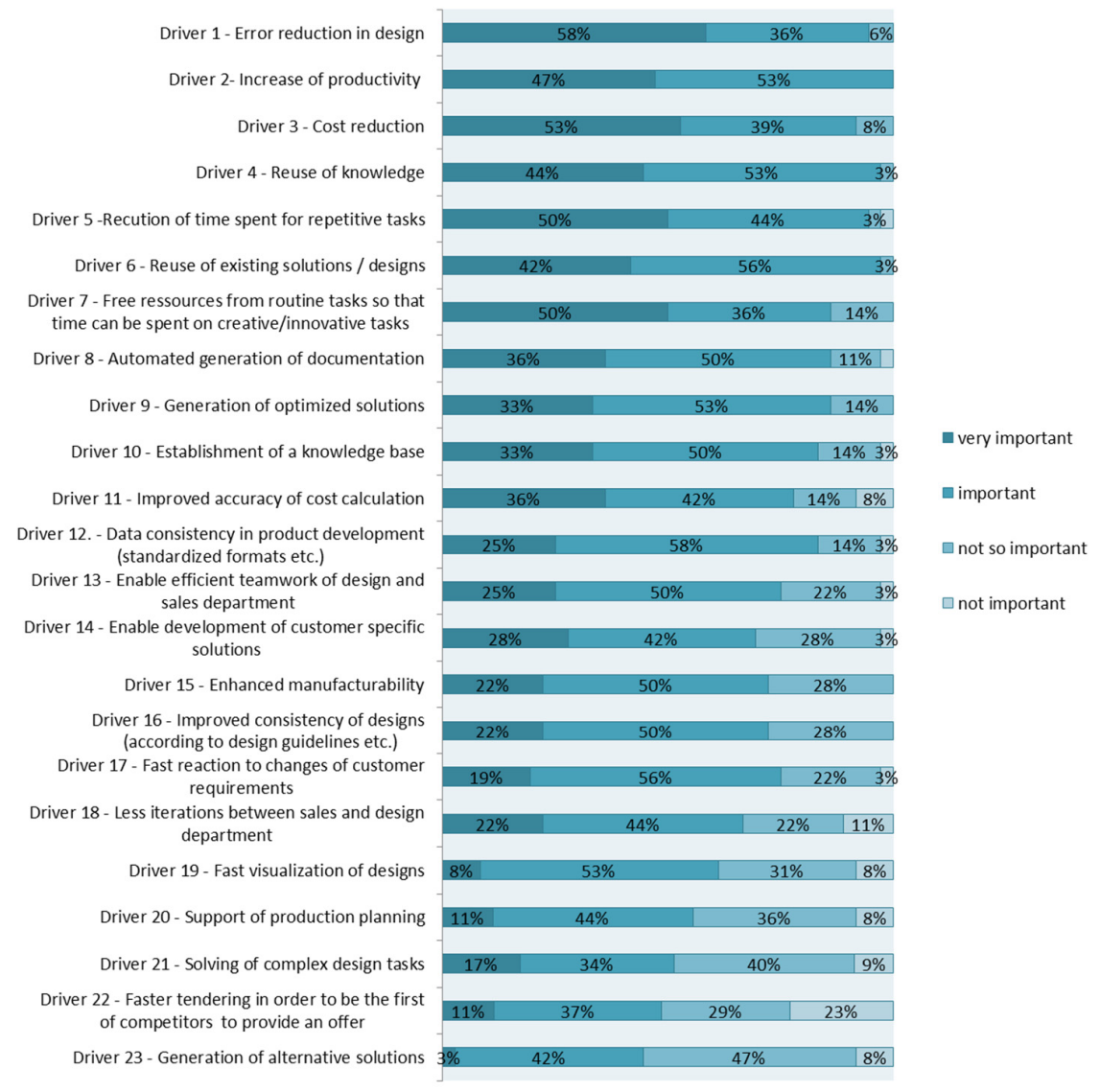

Figure 7. What is the relevance of the following drivers for design automation implementation? $(\mathbf{N}=\mathbf{3 6})$

\subsection{Network analysis}

The following subsections describe the network generation as performed for the data sets related to each of the hypotheses listed in Section 2.1. Details for the generation of networks are explained, i.e. which data corresponds to vertices, what type of edges is used and how edges are computed. For each network, the "SpringElectricalEmbedding" method was applied as described in Section 3.3.

\subsubsection{Data analysis for hypothesis 1}

In order to graphically illustrate how design automation knowledge, design automation experience and company size are interrelated, Figure 8 shows the network of participants. Thereby, vertices correspond to the participants and edges are created for the case that two participants provided the same answers to questions 7 and 8 , i.e. whether they have design automation knowledge or design automation experience, respectively. The vertex size is calculated in relation to the number of employees of the participant's company: the bigger the vertex the more employees there are. It can be seen that 3 distinct clusters are formed: on the left hand side the cluster of participants that both have heard about design automation and also have gained experience through application; the centre cluster with participants that have heard about design automation but have not gained any experience so far and the cluster on the right-hand side which indicates the participants that did not report any knowledge on design automation. 


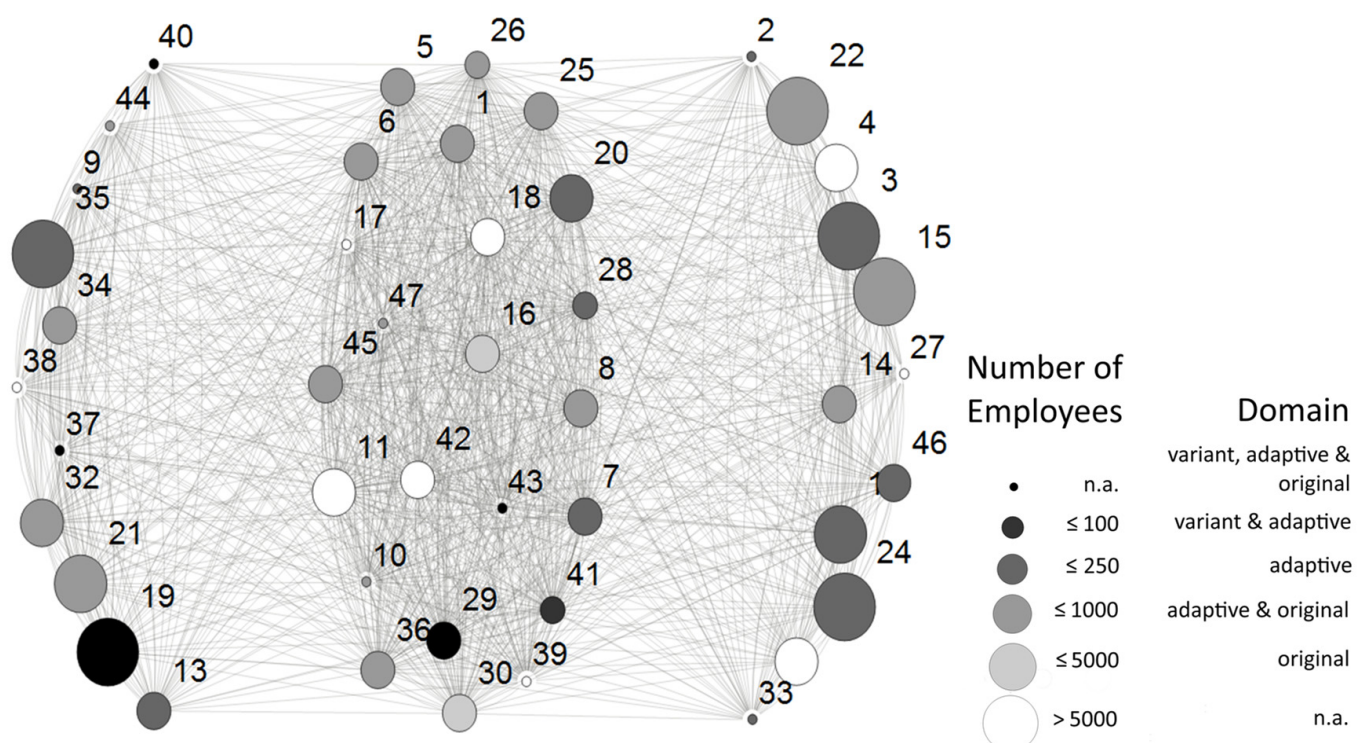

Figure 8. Network of responses whether experience of DA application exists. Cluster left: Participants with DA experience; central cluster: Participants have heard about DA but no experience; cluster right: No knowledge on DA

\subsubsection{Data analysis for hypothesis 2}

Similarly, Figure 9 shows the network of participants, i.e. vertices correspond to the participants of the survey. However, with respect to hypothesis 2, edges are generated for the case that two participants either apply the same types of design (question 1) and either they have the same level of design automation experience (question 8).

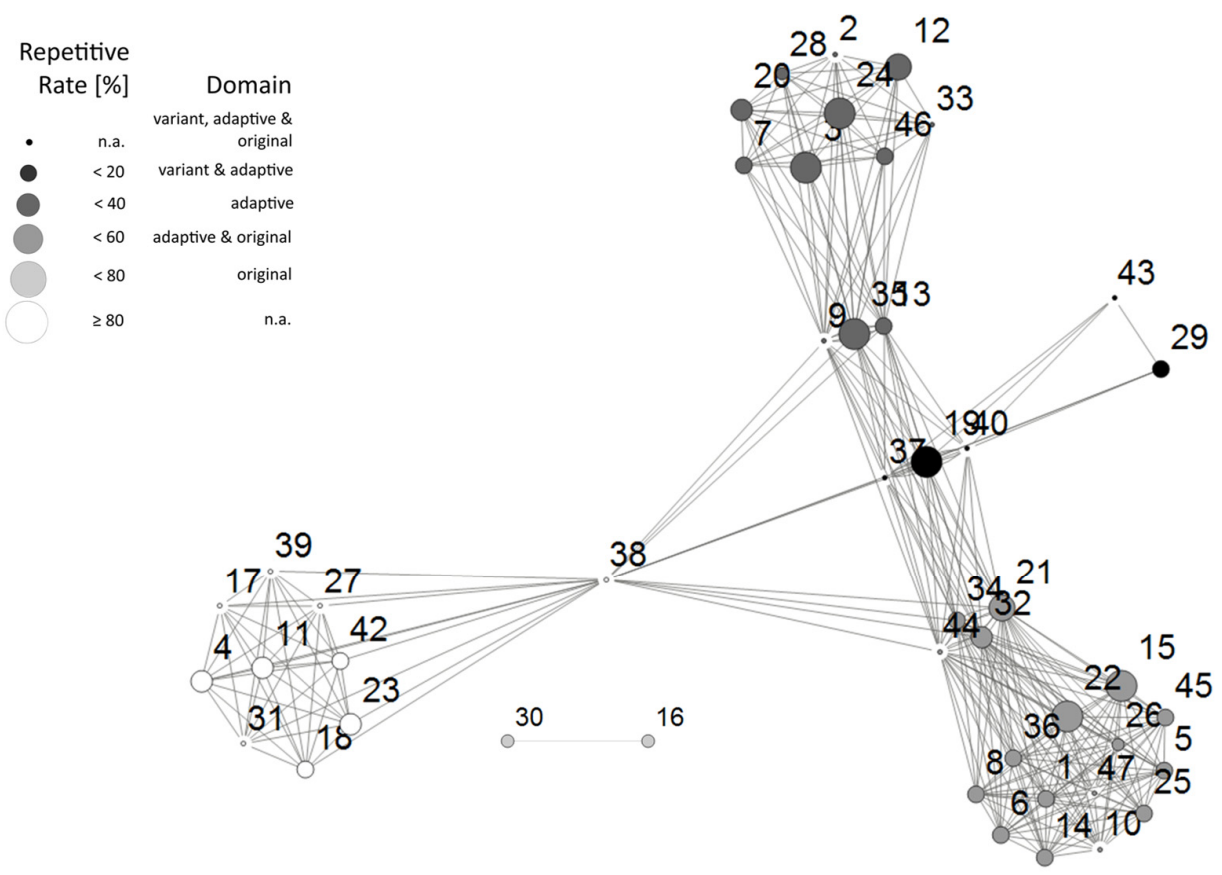

Figure 9. Network of type of product vs. design automation experience company size 
In order to better illustrate the results of the network generation, each type of design applied is indicated by a different grayscale value. Further, the vertex size is calculated in relation to the level of repetitiveness in product development i.e. \% of repetitive tasks as asked for in question 6 . The network highlights that clusters are formed based on the types of design; however, members are detached of the clusters to form a loosely linked central cluster for the case the participants have reported that they have already gained experience with design automation. For the companies exclusively applying original design, no experience with design automation is reported. That is why a remote cluster on the bottom with light grey vertices is formed.

\subsubsection{Data analysis for hypotheses 3 to 5}

Figure 10 illustrates how drivers and types of design applied are related based on weighted graphs. For each graph, the central vertex represents one specific type of design applied. The remainder of vertices in each graph correspond to the drivers. The shorter the distance between two vertices is, the higher is the rating of the participants for the specific driver. The number of data available for each graph is listed in the caption of each figure. Circles with colour coding are used to indicate the relative distance of the vertices from the central vertex - yellow: very high relevance; red: high relevance; blue: relevant; grey: not so relevant. Figure 10 shows three networks to visualize the rating of drivers of participants who state that their type of product design correspond to "adaptive", "adaptive \& original design" and "original design", respectively. For Figures 11, 12 and 13, the central vertex represents the rating of how many different disciplines are involved in design, the frequency of application of numerical simulations and whether design automation experience exists or not, respectively. For the sake of readability, the legend of drivers and description is provided on the next page.

\section{Discussion}

In the following, first the state of practice of companies with respect to design automation and the existing potential for further implementation of computational support in product development are discussed. Next, the current view of design automation is established by means of analysis of the rating of drivers from multiple perspectives, as shown in Figures 10 to 13. The networks are exploited for a more detailed analysis of correlations of data in order to finally reassert or weaken the list of hypotheses introduced in Section 3.1 and derive potential and opportunities for design automation application. Lastly, the pursued methodology is analysed and future work is indicated.

\subsection{Current state of design automation and corresponding potential}

Generally, there is large potential for further digitization of product development, on the one hand from the data management perspective, as shown in Figure 4(a), and on the other hand from a design automation point of view, as depicted in Figure 4(b). Regarding the latter, the vast majority of participants have already heard about the design automation, however, only $25 \%$ have actual experience with its application (cf. Figure 3). Interestingly, Figure 8 shows that designers from large companies are the ones that are more likely to have experience with design automation, but also the ones who are likely to have no knowledge at all about it. A reason could be that large organizations usually have departments concerned with IT support of product development, which also make the interface with software vendors. Thus, designers employed at large companies might not receive latest information from software vendors and are usually not directly involved in the specification and implementation of design automation solutions. A potential means to support designers is the development of methods to enable designers to identify and specify design automation task themselves (Vosgien et al., 2017). Considering knowledge about design automation, interestingly, most SMEs have knowledge about design automation and occasionally, design automation is also applied in SMEs, as depicted in Figure 8. Hence, hypothesis 1 needs to be weakened.

With respect to software tools for supporting design, it can be seen within Figure 2 that the majority of participants use CAD and ERP systems. Reason for the comparably low rate of usage of tools like CRM or CAPP might be the trend towards integration of capabilities in larger, monolithic systems, often referred to as "PLM systems" (Bilello, 2011). 

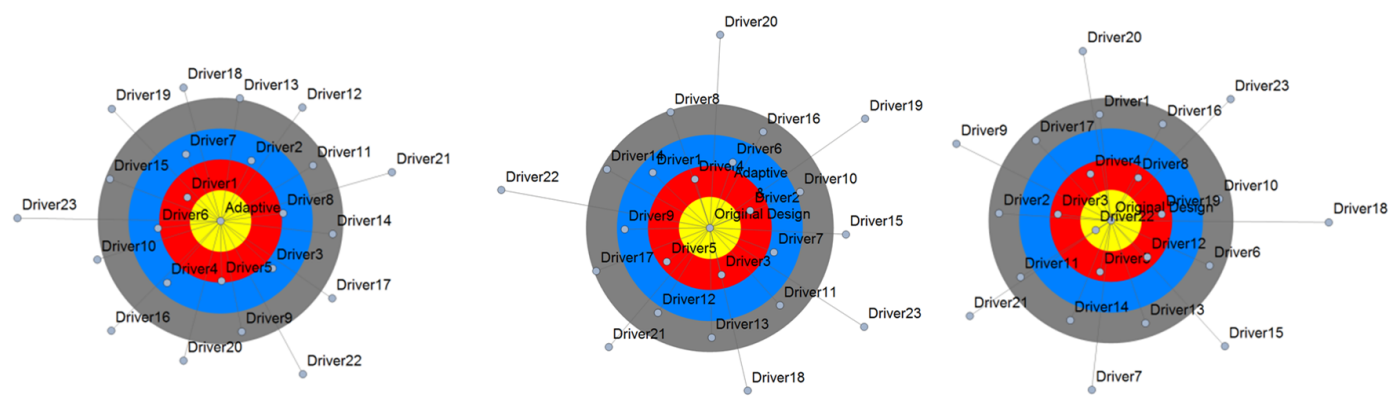

Figure 10. Type of design vs. design automation drivers: Adaptive Design (left, $\mathbf{N}=$ 12); Adaptive \& Original Design (centre; $N=17$ ); Original design (right; $N=2$ )
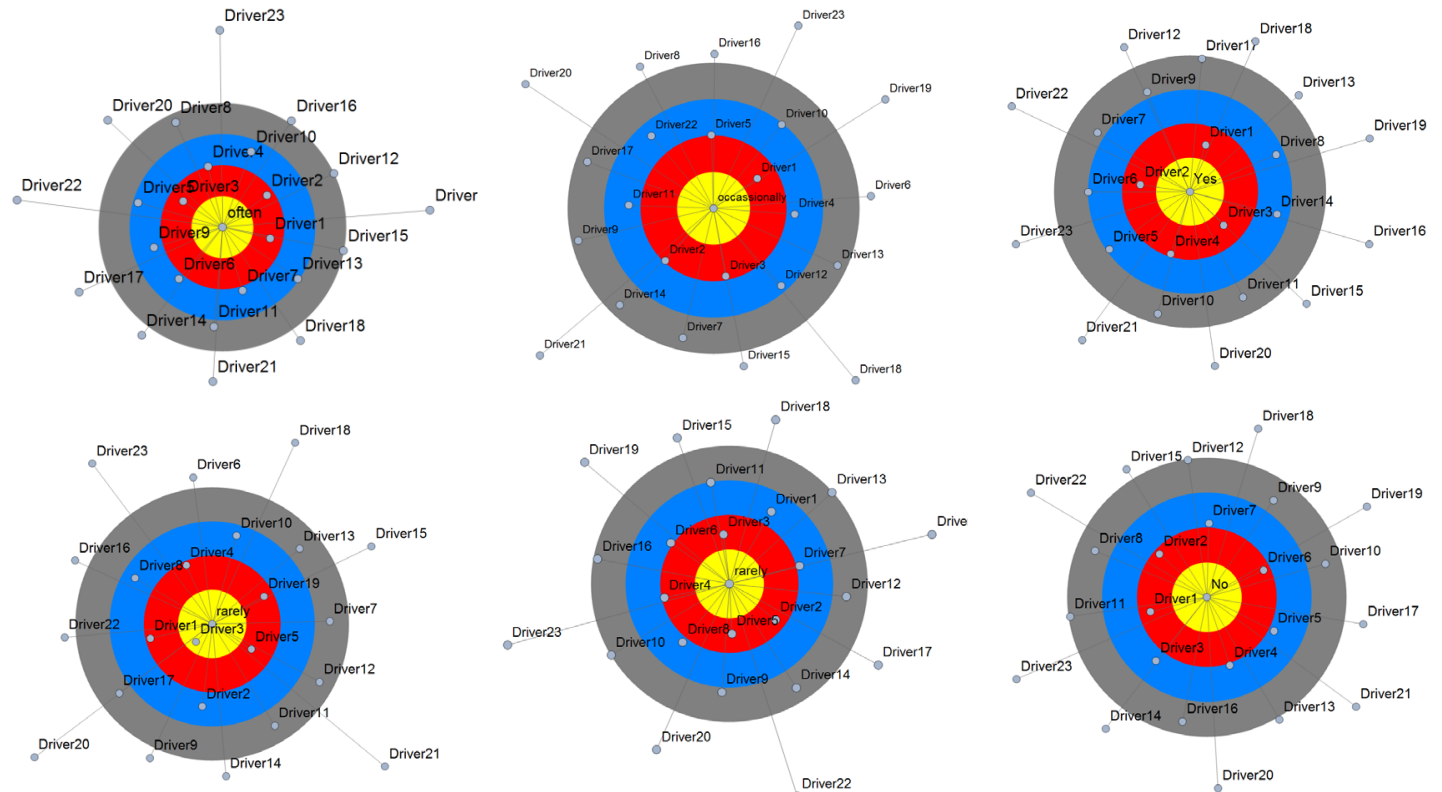

Driver 1 Error reduction in design

Driver 2 Increase of productivity

Driver 3 Cost reduction

Driver 4 Reuse of knowledge

Driver 5 Recution of time spent for repetitive tasks

Driver 6 Reuse of existing solutions / designs

Driver 7 Free ressources from routine tasks so that time can be

Driver 8 Automated generation of docum entation

Driver 9 Generation of optimized solutions

Figure 11.

Rating of multidisciplinarity of processes/tasks vs. design automation drivers:

Often (top; $N=14$ ); Rarely (bottom; $N=4$ )

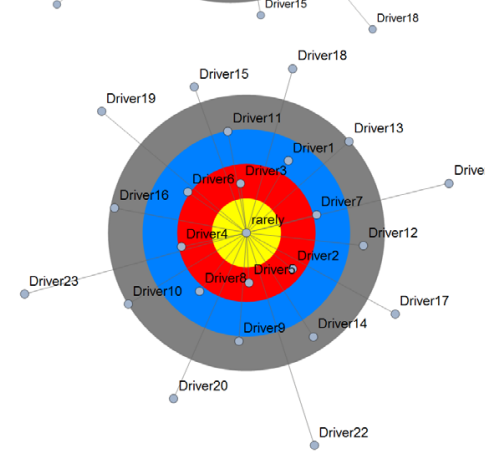

Driver 10 Establishment of a knowledge base

Driver 11 Improved accuracy of cost calculation

Driver 12 Data consistency in product development (standardized formats etc.)

Driver 13 Enable efficient teamwork of design and sales department

Driver 14 Enable development of customer specific solutions

Driver 15 Enhanced manufacturability

Driver 16 Improved consistency of designs (according to design guidelines etc.)

Figure 12.

Application frequency of numerical simulations vs.

design automation drivers: Occasionally

(top; $\mathrm{N}=7$ );

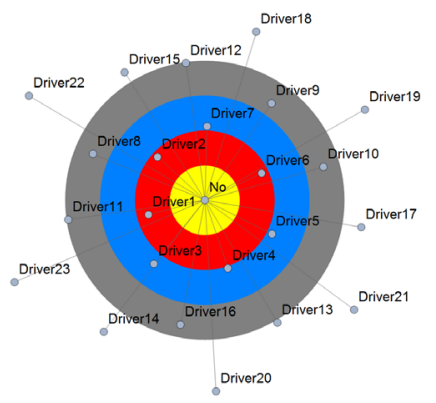

Driver 17 Fast reaction to changes of customer requirements

Driver 18 Less iterations betw een sales and design department

Driver 19 Fast visualization of designs

Driver 20 Support of production planning

Driver 21 Solving of com plex design tasks

Driver 22 Faster tendering in order to be the first of competitors to provide an offer

Driver 23 Generation of altemative solutions

Figure 13.

Design automation experience vs. design automation drivers: Yes (top; $N=11$ ); No (bottom; $\mathbf{N}=35$ )

Further, in Figure 2 it can be seen that only a minority of participants has deployed PDM systems which are crucial for workflow (Gerhard, 2016) and product configuration management (Mesihovic and 
Malmqvist, 2000). Most likely, its application is hindered by a lack of standards and maturity of processes which is needed for PDM deployment but also considered prerequisite for the application of design automation (Willner et al., 2016). Hence, the lack of maturity of processes and products might be a reason for the low rate of application of design automation in industry. With respect to applied types of design, Figure 1 shows that the majority of participants apply adaptive design within their product development, i.e. the reuse of existing design and modules and adaptations so to meet the customers' requirements. Figure 9 indicates that all the clusters associated to adaptive design have at least one participant who claims experience with design automation. On the other hand, participants who exclusively focus on original design have no experience with design automation. Thus, a detached cluster appears within Figure 9. Consequently, hypothesis 2 can be strengthened: design automation is currently more likely to be applied for tasks in adaptive design. With respect to the rate of repetitiveness, no conclusion can be drawn about the repetitiveness of automated tasks since the relevant participants did not answer this question.

\subsection{Design automation needs}

Figure 7 shows the ranked list of industrial drivers as rated by 36 participants of the online survey and it shows that drivers usually associated to the application of KBE are predominant, e.g. "error reduction" and "increase of productivity". On the other hand, the drivers associated to the implementation of CDS solutions (Rigger et al., 2016), i.e. generation of design alternatives, solving complex tasks are considered less relevant.

Considering hypothesis 3, it can be seen within in Figure 10 that the type of design impacts the rating of drivers: in comparison to participants applying adaptive design, participants that apply original design have potentially a higher need for the fast visualizations of design as well as a need for faster tendering. Instead, they do not consider important neither the optimization of designs neither the automation of routine tasks. Reason for this might be that the rate of routine tasks is substantially lower for original design than for adaptive design (cf. Figure 9). The need for visualization and support for tendering are most likely related to the large efforts usually spent in the conceptual design phase as performed in original design when visualization and detailed calculation of offers are simply not possible. In particular, CDS methods can support this stage of the design process by means of systematic investigation of the design space and thereby contribute to a more efficient conceptual design. However, these methods are still not perceived as key enablers and it is reflected in the rating of the drivers. Thus, the necessity to make the designers aware of CDS methods is urged.

With respect to hypothesis 4, Figure 11 shows that there is an impact of the multidisciplinary aspects of design on the rating of drivers. For the case "multiple disciplines are often involved", the rating corresponds to the one represented in Figure 7, however, if "no other discipline is involved" in design, reuse of designs as wells as optimization are considered less relevant. This is potentially due to a lower complexity of products and processes, thus, the less the need for systematics in design as well as application of design automation. Similarly, this is the case for participants who apply numerical simulations occasionally within product development and do not consider the reuse of existing designs relevant, as it can be seen in Figure 12. For participants who apply "numerical simulations rarely", it is interesting to note that cost reduction and reduction of repetitive tasks is considered more important than error reduction. Thus a need for design automation application to automate pre- and post-processing of designs could be derived. Altogether, the findings with respect to product complexity reassert hypothesis 4 that states that the more complex the product, the more important it is to reduce errors in design. With respect to design automation experience of participants, it can be seen in Figure 13 that for the case that experience has already been gained, the application of design automation for development of customer specific solutions is gaining importance at the cost of drivers related to consistency of designs, reduction of iterations in the sales processes and faster visualization of designs, the latter ones being usually associated to benefits of sales configurators (Willner et al., 2016). Reasons could be unfulfilled expectations on the one hand, and the acknowledgement of potential for coping with fast changing requirements in product development on the other hand. Therefore, with respect to hypothesis 5, instead

of a more enthusiastic point of view of design automation, the results of the survey rather suggest a change in priorities. 
Summing up, from the rating of drivers it can be seen that KBE drivers are still predominant and the potential CDS methods are currently not recognised as design automation potential, even not for the participants focussing on original design or multidisciplinary design. Hence, methods and tools are needed in order to enable designers to identify and define design automation tasks themselves to better communicate opportunities to practitioners. Also, both design education and software vendors need to be involved to advocate and highlight the potential design automation offers to further strengthen the application of design automation in industry. However, from a process and product maturity point of view it can be questioned how much the industry is prepared for the application of design automation, so that computational support can be directly integrated in existing workflows and IT environments. However, with this respect, additional investigation of state of practice of design process formalization and product characteristics is needed by means of a follow-up survey. At the same time, reliability of the questionnaire (Robinson, 2016) could be supported by means of additional verification of participant's answers by means of asking related follow up questions in the context of design automation as well as the execution of statistical tests for verification of hypotheses.

\section{Conclusions}

This paper contributes by means of a systematic investigation of the state of practice of design automation and establishment of the practitioners' view of the topic by means of an online survey. This works extends existing state-of-practice reviews by means of a hypothesis driven multivariate analysis based on network analyses which allows the systematic investigation of interrelations in the data from the survey. Further, this study contributes through validation of industrial drivers with respect to design automation, in particular with the ones frequently mentioned by the CDS community. Whereas a large potential for computational support of product development is highlighted, it is shown that designers still do not acknowledge the value of design automation methods to support the early design phases by means of solutions space exploration and solving of complex design tasks. Instead, the point of view to consider design automation as a means to reduce repetitive activities and save time in the latter stages of product development is predominant. Reason for this might be stemming from the lack of knowledge about the opportunities state of the art design automation offers. Thus, there remains much hidden potential which can be released by making practitioners aware of the availability of methods that support ideation and automation of complex design tasks. First, the need for methods and tools that enable designer to identify and define design automation tasks themselves is highlighted so to change the perception of design automation in industry (Bolognini et al., 2012). Second, both the design education and software vendors need to be involved in order to advocate and communicate the potential the entire spectrum of design automation offers. Future work comprises the investigation of impacts of product and process maturity as prerequisite for implementation of computational support in design by means of an extended survey as well as additional systematic in verification of hypotheses by means of statistical tests.

\section{Acknowledgment}

This work was supported by the K-Project 'Advanced Engineering Design Automation' (AEDA) that is financed under the COMET (COMpetence centers for Excellent Technologies) funding scheme of the Austrian Research Promotion Agency as well as by the project 'Automation Studio for Innovative Design' (ASID) that is financed under the COIN "Aufbau" funding scheme of the Austrian Research Promotion Agency.

Special thanks go to Roman Dorfmayr, MSc and FH-Hon.Prof. Dr. Dr. Herwig Schneider from the "Industriewissenschaftliches Insitut - IWI" for support in design, execution and evaluation of the survey.

\section{References}

Bilello, P. (2011), PLM \& System Overlaps. [online] CIM Data.

Blessing, L.T.M. and Chakrabarti, A. (2009), DRM, a design research methodology, Springer, London. https://doi.org/10.1007/978-1-84882-587-1

Bolognini, F., Jauregui Becker, J.M. and Schotborch, W.O. (2012), “An Investigation into Limited Integration of Computational Design Synthesis in Common Design Practice", Proceedings of DESIGN $2012 /$ the $12^{\text {th }}$ International Design Conference, Dubrovnik, Croatia, May 21-24, 2012, pp. 727-736. 
Cederfeldt, M. and Elgh, F. (2005), "Design automation in SMEs-current state, potential, need and requirements", Proceedings of ICED'05 / the $15^{\text {th }}$ International Conference on Engineering Design, Melbourne, Australia, August 15-18, 2005.

Chandrasegaran, S.K., Ramani, K., Sriram, R.D., Horváth, I., Bernard, A. et al. (2013), "The evolution, challenges, and future of knowledge representation in product design systems", Computer-Aided Design, Vol. 45 No. 2 , pp. 204-228. https://doi.org/10.1016/j.cad.2012.08.006

Dassault Systems (2010), Using Design Automation to Reduce Costs, increase profitability, Dassault Systems, SolidWorks Corp., Concord, USA.

Gerhard, D. (2016), "Daten- und Informationsmanagement PDM/PLM", In: Lindeman, U. (Ed.), Handbuch Produktentwicklung, Carl Hanser Verlag, München, pp. 215-246. https://doi.org/10.3139/9783446445819

Hvam, L., Pape, S. and Nielsen, M.K. (2006), "Improving the quotation process with product configuration", Computers in Industry, Vol. 57 No. 7, pp. 607-621. https://doi.org/10.1016/j.compind.2005.10.001

Mesihovic, S. and Malmqvist, J. (2000), "Product data management (PDM) system support for the engineering configuration process", Proceedings of ECAI $2000 /$ the $14^{\text {th }}$ European Conference on Artificial Intelligence, Berlin, Germany, August 20-25, 2005.

Pahl, G., Beitz, W., Feldhusen, J. and Grote, K.H. (2007), Engineering Design: A Systematic Approach, Springer, London. https://doi.org/10.1007/978-1-84628-319-2

Quigley, A.J. (2001), Large Scale Relational Information Visualization, Clustering, and Abstraction, PhD thesis, The University of Newcastle, Newcastle.

Rigger, E., Münzer, C. and Shea, K. (2016), "Estimating the Potential of State of the Art Design Automation Tasks, Methods, and Benefits", Proceedings of DESIGN 2016 / the $14^{\text {th }}$ International Design Conference, Dubrovnik, Croatia, May 16-19, 2016, The Design Society, Glasgow, pp. 421-432.

Rigger, E., Stanković, T. and Shea, K. (2018), “Task Categorization for Identification of Design Automation Opportunities", Journal of Engineering Design, Vol. 29 No. 3, pp. 131-159. https://doi.org/10.1080/09544828.2018.1448927

Robinson, M.A. (2016), "Quantitative Research Principles and Methods for Human-Focused Research in Engineering Design”, In: Cash, P., Stanković, T. and Štorga, M. (Eds.), Experimental Design Research, Springer, Cham, pp. 41-64. https://doi.org/10.1007/978-3-319-33781-4_3

Shephard, M.S., Beall, M.W., O’Bara, R.M. and Webster, B.E. (2004), “Toward simulation-based design”, Finite Elements in Analysis Design, Vol. 40 No. 12, pp. 1575-1598. https://doi.org/10.1016/j.finel.2003.11.004

Tanur, J.M. (2011), "Margin of Error", In: Lovric, M. (Ed.), International Encyclopedia of Statistical Science, Springer, Berlin, Heidelberg, pp. 765-765. https://doi.org/10.1007/978-3-642-04898-2 34

Verhagen, W.J.C., Bermell-Garcia, P., van Dijk, R.E.C. and Curran, R. (2012), “A critical review of KnowledgeBased Engineering: An identification of research challenges", Advanced Engineering Informatics, Vol. 26 No. 1, pp. 5-15. https://doi.org/10.1016/j.aei.2011.06.004

Vosgien, T., Rigger, E., Schwarz, M. and Shea, K. (2017), “A Federated Enterprise Architecture and MBSE Modeling Framework for integrating Design Automation into a global PLM approach", Proceedings of the $14^{\text {th }}$ IFIP WG 5.1 International Conference, PLM 2017, Sevilla, Spain, July 10-12, 2017, Springer, Cham, pp. 36-48. https://doi.org/10.1007/978-3-319-72905-3 4

Willner, O., Gosling, J. and Schönsleben, P. (2016), "Establishing a maturity model for design automation in salesdelivery processes of ETO products", Computers in Industry, Vol. 82, pp. 57-68. https://doi.org/10.1016/j.compind.2016.05.003

Zhang, L.L. (2014), "Product configuration: a review of the state-of-the-art and future research", International Journal of Production Research, Vol. 52 No. 21, pp. 6381-6398. https://doi.org/10.1080/00207543.2014.942012

Eugen Rigger, Doctoral Student

V-Research GmbH, Design Automation

Stadtstraáe 33, 6850 Dornbirn, Austria

Email: eugen.rigger@v-research.at 